\title{
The Complex Dynamics of Hepatitis B Infected Individuals with Optimal Control*
}

\author{
DIN Anwarud · LI Yongjin · SHAH Murad Ali
}

DOI: $10.1007 / \mathrm{s} 11424-021-0053-0$

Received: 19 March 2020 / Revised: 12 June 2020

(C)The Editorial Office of JSSC \& Springer-Verlag GmbH Germany 2021

\begin{abstract}
This paper proposes various stages of the hepatitis B virus (HBV) besides its transmissibility and nonlinear incidence rate to develop an epidemic model. The authors plan the model, and then prove some basic results for the well-posedness in term of boundedness and positivity. Moreover, the authors find the threshold parameter $R_{0}$, called the basic/effective reproductive number and carry out local sensitive analysis. Furthermore, the authors examine stability and hence condition for stability in terms of $R_{0}$. By using sensitivity analysis, the authors formulate a control problem in order to eradicate HBV from the population and proved that the control problem actually exists. The complete characterization of the optimum system was achieved by using the $4^{\text {th }}$-order Runge-Kutta procedure.
\end{abstract}

Keywords Hepatitis B epidemic model, non-linear incidence, normalized sensitivity index, numerical simulation, optimization theory, reproduction number, stability analysis.

\section{Introduction}

The study of modeling is one of the influence instrument to understand the time dynamics of various infectious diseases. Numerous of biologists and mathematicians have analyzed mathematical models for the spreading of communicable infections in community ${ }^{[1-12]}$. HBV infection is one of the severe health problem which caused million of people suffered. Approximately 0.78 million individuals dies each year from the consequences of $\mathrm{HBV}^{[13]}$.

It can be transferred by different ways, which include syringes sharing, transfusion of blood and sexual interactions, etc ${ }^{[1,6]}$. HBV infection also transfers maternally, i.e., from infected mother to baby, which is known the vertical type of transmission. Utmost infected individual in the acute stage, the immune system have the capability to clear the HBV, however for some

DIN Anwarud · LI Yongjin (Corresponding author)

Department of Mathematics, Sun Yat-sen University, Guangzhou 510275, China.

Email: anwarud@mail.sysu.edu.cn; stslyj@mail.sysu.edu.cn.

SHAH Murad Ali

School of Mathematical Sciences; Beijing Normal University, Beijing 100875, China.

Email: muradmath@yahoo.com.

*This research was supported by the National Natural Science Foundation of China under Grant No. 11971493.

$\diamond$ This paper was recommended for publication by Editor YOU Keyou. 
one, especially children are particularly vulnerable and cultivate acute contaminations, which further leads to the chronic carrier and may develop liver cancer. While there are a vaccine and new methods to avoid the transmission. This disease has multiple stages, i.e., acute and chronic. The first one is the primary 6 months period, if some one is exposed. Chronic stage refers to the most serious stage of HBV infection in which serious problems may occur. This infection usually has no history regarding acute stage, but produce scarring of liver and as a result develop failure of liver ${ }^{[14]}$.

Different mathematician and biologists formulated epidemic models to forecast the dynamics of HBV ${ }^{[14-16]}$. The incidence rate has a vital and major role in studying the modeling of infectious diseases. In sexually transmittable diseases, e.g., HBV, HIV, etc., non-linear incidence is more realistic then other incidence rates. One of the type of incidence rate is bilinear incidence $\beta S I$, which was also frequently used ${ }^{[17-19]}$, where $\beta, S$ and $I$ characterize the interaction rate, the susceptible class, the infectious class respectively. Non-linear incidence rate, which is defined by $\frac{\beta S I}{N}$ used by many authors, see for detail ${ }^{[20,21]}$. In this rate, $\beta$ demonstrates the disease transfer rate, while $S, I$ and $N$ respectively show susceptible population, infected population and total population.

We investigate a model to study the dynamics of HBV transmission and formulate an control mechanism. The current study is actually divided into two main parts, the dynamic and control. In the dynamic part, we discuss and prove the stabilities of the proposed model, however in control part, we plan a control strategy to minimize the infection. More preciously, first, we prove the mathematical properties (boundedness, positivity) to show the well posed-ness and biological feasibility. The threshold quantity will be obtained to discuss the sensitivity analysis. We discuss stability at both equilibrium using the linearization, the Lyapunov theory and geometrical approach. We also investigate a hepatitis B virus elimination mechanism using three control variables (isolation, vaccination and treatment). The control strategy was formulated by using sensitivity of $R_{0}$ with respect to (w.r.t) parameters. Moreover, we obtain the optimality condition and discuss the existence of solution. Numerical results will be presented for supporting analytical findings. Finally, we conclude our work.

The organization of the paper is structured as follows: The proposed HBV model is given in Section 2. We deliberate the properties of existence, positive solution, boundedness and biologically feasibility in Section 3. The reproductive number and the equilibria are presented in Section 4. The stability and numerics of the stability results are given in Sections 5 and 6, respectively. In Section 7, we discussed the local sensitivity analysis. The formulation of the optimal control mechanism and its existence of positive solution with optimality condition are respectively presented in Subsections 8.1 and 8.2. Simulations are presented for the verification of the optimization in Section 9. Section 10 is devoted to concluding remarks.

\section{Model Formulation}

We present the model for HBV spreading with non-linear incidence. According to characteristic of the HBV imposing assumption given by: 
$a_{1}$. The entire population is shown by by $N(t)$ and is subdivided into 6 different subgroups of $S(t)$ (susceptible/vulnerable), $L(t)$ (exposed/latent), $A(t)$ (acute infected), $C(t)$ (chronic carrier infectious), $R(t)$ (recovered) and $V(t)$ (vaccinated), i.e., $S+A+L+C+V+R=N$, which oi changing w.r.t time $t$.

$a_{2}$. The entry of new born, which are parentally infected will go to carrier class.

$a_{3}$. The new born, which are not parentally infected will go to the susceptible class.

$a_{4}$. Successful vaccinated individuals go to the vaccinated class.

$a_{5}$. Removed population have immunity.

$a_{6}$. The new born with effective vaccination will go to vaccination class.

Thus, the above assumptions $a_{1}-a_{6}$ lead to the following model:

$$
\begin{aligned}
\frac{d S(t)}{d t}= & \xi b(1-\eta C(t)) N+\phi V(t)-\frac{\beta S(t) A(t)}{N}-\frac{\beta}{N} \gamma S(t) C(t) \\
& -\left(\gamma_{3}+d_{0}\right) S(t), \quad S(0)>0, \\
\frac{d L(t)}{d t}= & \frac{\beta A(t) S(t)}{N}+\frac{\gamma \beta C(t) S(t)}{N}-\left(\sigma+d_{0}\right) L(t), \quad L(0) \geq 0, \\
\frac{d A(t)}{d t}= & -\left(d_{0}+\gamma_{1}\right) A(t)+\sigma L(t), \quad A(0) \geq 0, \\
\frac{d C(t)}{d t}= & \xi b \eta C(t) N-\left(d_{1}+d_{0}+\gamma_{2}\right) C(t)+p \gamma_{1} A(t), \quad C(0) \geq 0, \\
\frac{d R(t)}{d t}= & \gamma_{1}(1-p) A(t)+\gamma_{2} C(t)-d_{0} R(t), R(0) \geq 0, \\
\frac{d V(t)}{d t}= & (1-\xi) b N-\left(\phi+d_{0}\right) V(t)+\gamma_{3} S(t), \quad V(0)>0 .
\end{aligned}
$$

Table 1 Parameters description of the proposed model (1)

\begin{tabular}{cl}
\hline Notation & Parameter description \\
\hline$b$ & birth rate \\
$\xi$ & fraction of new-born without effective immunization \\
$\phi$ & rate of vertically infected individual \\
$\beta$ & induced immunity rate with wane vaccination \\
$\gamma$ & reduced transmission rate \\
$d_{1}, d_{0}$ & disease induced and natural death rates \\
$\sigma$ & the rate at which people coming to acute class from \\
& the latent population \\
$\gamma_{1}$ & rate of acute to chronic \\
$\gamma_{2}$ & the rate at which people coming to recovered class \\
& from the carriers population \\
$\gamma_{3}$ & vaccination rate \\
$p$ & the rate at which recover people fails in acute class \\
\hline
\end{tabular}




\section{Fundamental Properties of the Model}

Regarding the fundamental properties of the proposed model (1), we shall prove the following results.

Proposition 3.1 The orthant $R_{+}^{6}$ is positively invariant for the proposed model presented in (1).

Proof Assume that $X=(S, L, A, C, R, V)^{\mathrm{T}}$, then the model (1) can be written as

$$
\frac{d X(t)}{d t}=G X+H
$$

where

$$
\begin{aligned}
G=\left(\begin{array}{cccccc}
-\frac{\beta A(t)}{N}-\frac{\gamma \beta C(t)}{N}-d_{0}-\gamma_{3} & 0 & -b \xi \eta N & 0 & 0 & \phi \\
\frac{\beta A(t)}{N}+\frac{\gamma \beta C(t)}{N} & -\left(d_{0}+\gamma_{1}\right) & 0 & 0 & 0 & 0 \\
0 & \sigma & -\left(d_{0}+\gamma_{1}\right) & 0 & 0 & 0 \\
0 & 0 & \gamma_{1}(1-p) & \gamma_{2} & -d_{0} & 0 \\
-Y_{11} & 0 & 0 & 0 & 0 & 0 \\
\gamma_{3} & 0 & 0 & 0 & 0 & -\left(d_{0}+\phi\right)
\end{array}\right), \\
H=\left(\begin{array}{c}
b \xi N \\
0 \\
0 \\
0 \\
0
\end{array}\right)
\end{aligned}
$$

Clearly matrix $H \geq 0$. Also the off-diagonals of $G$ are nonnegative, which shows that $G$ is Metzler matrix. Thus, the system (1) is positively invariant in $R_{+}^{6}$.

Proposition 3.2 If $t>0$ along with non-negative initial condition, the solutions of the model (1) are positive, if exist.

Proof Let $I \subset[0,+\infty)$. If the solution of the system (1) exists in $I$, then the solution of the 1 st equation in the system (1) look like

$$
\begin{aligned}
S(t)= & \mathrm{e}^{-\left(\gamma_{3}+d_{0}\right) t-\int_{0}^{t} \frac{1}{N}[\beta A(x)+\beta \gamma C(x)] d x}\left[S(0)+\int_{0}^{t} \mathrm{e}^{\left(\gamma_{3}+d_{0}\right) \tau+\int_{0}^{\tau} \frac{1}{N}[\beta A(w)+\beta \gamma C(w)] d w}\right. \\
& \times(b \xi N(1-\eta C(\tau))+V(\tau) \phi) d \tau] .
\end{aligned}
$$

From Equation (5) it is noted that $S(t)>0$ only if $A(t)$ and $C(t)$ are positive. From the third equation of the model, we can write

$$
A(t)=\mathrm{e}^{-\left(\gamma_{1}+d_{0}\right) t} A(0)+\mathrm{e}^{-\left(\gamma_{1}+d_{0}\right) t} \int_{0}^{t} \sigma L(\tau) \mathrm{e}^{\left(\gamma_{1}+d_{0}\right) \tau} d \tau
$$


which shows that $A(t) \geq 0$ whenever $L(t) \geq 0$. Similarly, positivity of $C(t)$ depends on the positivity of $A$. In the same way, it can be investigated that the other classes $R(t)$ and $V(t)$ are nonnegative, which proves the conclusion.

Remark 3.3 The proposed model (1) is a dynamical system in the following region given by

$$
\Omega=\left\{(S, L, A, C, R, V) \in R_{+}^{6}: N(t) \leq \frac{b N}{d_{0}}\right\} .
$$

\section{Threshold Parameter (Basic Reproductive Number) and Equilibria}

This section of the manuscript is devoted to finding of the threshold parameter (basic reproductive number) and equilibria of the model.

\subsection{Computation of Equilibria and Basic Reproduction Number}

The model under consideration (1) has a DFE point denoted by $F_{0}=\left(S_{0}, 0,0,0,0, V_{0}\right)$, where

$$
S_{0}=\frac{N b\left(\phi+\xi d_{0}\right)}{d_{0}\left(d_{0}+\phi+\gamma_{3}\right)}, \quad V_{0}=\frac{N b\left(d_{0}-d_{0} \xi+\gamma_{3}\right)}{d_{0}\left(d_{0}+\phi+\gamma_{3}\right)} .
$$

In epidemic models, the threshold parameter $R_{0}$ has a vital role and is a significant approach. It characterizes the average amount of newly infections. We follow the techniques of DriesscheWatmough $^{[22,23]}$. Let $\chi=(L, A, C)^{\mathrm{T}}$, then by the use of

$$
\bar{F}=\left(\begin{array}{c}
\frac{\beta}{N} S(t) A(t)+\frac{\beta \gamma C(t) S(t)}{N} \\
0 \\
0
\end{array}\right), \quad \bar{W}=\left(\begin{array}{c}
\left(d_{0}+\sigma\right) L(t) \\
\left(\gamma_{1}+d_{0}\right) A(t)-\sigma L(t) \\
\left(d_{1}+d_{0}+\gamma_{2}-b \xi \eta N\right) C(t)-p \gamma_{1} A(t)
\end{array}\right),
$$

the model (1), yields

$$
\frac{d \chi}{d t}=\bar{F}-\bar{W}
$$

where $\bar{W}$ and $\bar{F}$ are the matrices containing the linear and nonlinear terms, respectively. Let $q_{2}=d_{0}+\sigma, q_{3}=\gamma_{1}+d_{0}, q_{4}=-b \xi \eta N+\gamma_{2}+d_{1}+d_{0}$ and taking the Jacobian of Equation (9) at DFE point $F_{0}=\left(S_{0}, 0,0,0,0, V_{0}\right)$, which becomes the following

$$
F=\left(\begin{array}{ccc}
0 & \frac{\beta}{N} S_{0} & \frac{\beta \gamma}{N} S_{0} \\
0 & 0 & 0 \\
0 & 0 & 0
\end{array}\right), \quad W=\left(\begin{array}{ccc}
q_{2} & 0 & 0 \\
-\sigma & q_{3} & 0 \\
0 & -p \gamma_{1} & q_{4}
\end{array}\right)
$$

The dominant eigenvalue $\rho$, of $\bar{K}=F W^{-1}$ is the threshold quantity, $R_{0}$. We find $R_{0}$, for the model (1), which has the form, $R_{0}=\gamma_{01}+\gamma_{02}$ with

$$
\gamma_{01}=\frac{\sigma \beta S_{0}}{N q_{2} q_{3}}, \quad \gamma_{02}=\frac{\sigma \beta S_{0} \gamma \gamma_{1} p}{N q_{2} q_{3} q_{4}}, \quad \text { where } S_{0}=\frac{b N\left(d_{0}+\phi\right)}{d_{0}\left(d_{0}+\phi+\gamma_{3}\right)} .
$$


Similarly endemic-state of the system (1) is represented by $F_{\star}=\left(S_{*}, L_{*}, A_{*}, C_{*}, R_{*}, V_{*}\right)$ exist, if $R_{0}>1$, where

$$
\begin{aligned}
S_{*} & =\frac{q_{2} q_{3} q_{4}}{q_{4}+p \gamma \gamma_{1}}, \quad L_{*}=\frac{d_{0} q_{2} q_{3}^{2} q_{4}\left(\gamma_{3}+q_{5}\right)\left(q_{4}-b \xi \eta N\right)\left(R_{0}-1\right)}{q_{5} \sigma\left(p \gamma \gamma_{1}+q_{4}\right)\left(\left(q_{4}+p \gamma \gamma_{1}\right) \beta S_{*}+b \xi \eta p \gamma_{1}\right)}, \\
A_{*} & =\frac{d_{0} q_{2} q_{3} q_{4}^{2}\left(q_{5}+\gamma_{3}\right)\left(R_{0}-1\right)}{q_{5}\left(\gamma_{1} p \gamma+q_{4}\right)\left(\left(\gamma_{1} p \gamma+q_{4}\right) S_{*} \beta+\xi b N \eta \gamma_{1} p\right)}, \\
C_{*} & =\frac{\gamma p \beta d_{0} q_{3} \gamma_{1}\left(q_{5}+\gamma_{3}\right)\left(R_{0}-1\right) S_{*}^{2}}{q_{5}\left(\left(\gamma_{1} p \gamma+q_{4}\right) \beta S_{*}+\xi b N \eta \gamma_{1} p\right)}, \quad R_{*}=\frac{1}{d_{0}}(1-p) \gamma_{1} A_{*}+\gamma_{2} C_{*}, \\
V_{*} & =\frac{1}{q_{5}}\left((1-\xi) b N+\gamma_{3} S_{*}\right),
\end{aligned}
$$

$q_{1}=d_{0}+\gamma_{3}$ and $q_{5}=d_{0}+\phi$. Clearly, there does not exist a positive endemic-state, if $R_{0}<1$, but the case of $R_{0}>1$, implies the existence of a unique positive equilibrium.

\section{$5 \quad$ Stability Analysis}

Usually, epidemic models are used to exhibiting the long-term behavior of an infectious disease. For this purpose, researchers are using the tools of stability analysis as described in [24-27]. To discuss the stability analysis of the proposed model, we prove the following subsequent results.

Remark 5.1 If we assume that $R_{0}<1$, then the DFE of the model is stable locally asymptotically, while unstable, whenever $R_{0}>1$.

Theorem 5.2 The DFE point $F_{0}$ is stable globally asymptotically, if $R_{0}<1$, while unstable, if $R_{0}>1$.

Proof Assume that $\psi(t)=\left(S-S_{0}\right)+L(t)+A(t)+C(t)+R(t)+\left(V-V_{0}\right)$ and suppose the function defined by

$$
\Gamma(t)=\frac{1}{2}[\psi(t)]^{2}+k_{1}\left(S-S_{0}\right)+k_{2} L(t)+k_{5}\left(V-V_{0}\right)+k_{3} A(t)+k_{4} C(t),
$$

where the positive constants $k_{i}$ for $i=1,2, \cdots, 5$ will be determined later. Differentiating Equation (14) and using the system (1), we get

$$
\begin{aligned}
\frac{d \Gamma(t)}{d t}= & (\psi(t))\left(b N-d_{0} N(t)-d_{1} C(t)\right) \\
& +k_{1}\left(\phi V(t)+\xi N b(1-\eta C(t))-\frac{\beta A(t) S(t)}{N}-\frac{\beta \gamma C(t) S(t)}{N}-\left(\gamma_{3}+d_{0}\right) S(t)\right) \\
& +k_{2}\left(\frac{\beta S(t) A(t)}{N}+\frac{\gamma \beta S(t) C(t)}{N}-\left(\sigma+d_{0}\right) L(t)\right)+k_{3}\left(-\left(\gamma_{1}+d_{0}\right) A(t)+\sigma L(t)\right) \\
& +k_{4}\left(\xi b \eta N C(t)+\gamma_{1} p A(t)-\left(d_{1}+\gamma_{2}+d_{0}\right) C(t)\right) \\
& +k_{5}\left((1-p) \gamma_{1} A(t)+\gamma_{2} C(t)-d_{0} R(t)\right)+k_{6}\left((1-\xi) N b-\left(d_{0}+\phi\right) V(t)+\gamma_{3} S(t)\right) .
\end{aligned}
$$


By assigning the values of $q_{2} q_{4}$ to $K_{i}$ where $i=1,2,3,5$ and $k_{4}=\sigma \beta \gamma S_{0}$, then Equation (15) becomes

$$
\begin{aligned}
\frac{d \Gamma(t)}{d t}= & -(\psi(t))^{2}-(\psi(t)) d_{1} C(t) \\
& -\xi b N \eta\left(d_{0} \sigma\right)\left(d_{1}+\gamma_{2}+d_{0}-\xi b N \eta\right) C(t) \\
& -d_{0}\left(d_{0}+\sigma\right)\left(d_{0}+\gamma_{2}+d_{1}-\xi b \eta N\right) L(t) \\
& -\left(d_{0}+\sigma\right)\left(d_{0}+\gamma_{1}\right)\left(d_{0}+\gamma_{2}+d_{1}-\xi b N \eta\right)\left(1-\gamma_{02}\right) \\
& -\beta \sigma \gamma\left(d_{0}+\gamma_{2}+d_{1}-\xi b N \eta\right) S_{0} C(t) \\
& -\left(d_{0}+\sigma\right)\left(d_{0}+\gamma_{2}+d_{1}-\xi b N \eta\right)\left(\left(S-S_{0}\right) d_{0}+\left(V-V_{0}\right)\right) .
\end{aligned}
$$

Equation (16) gives that, if $R_{0}<1$, then $\frac{d \Gamma(t)}{d t}<0$. Also $\frac{d \Gamma(t)}{d t}=0$, at the DFE point, therefore, LaSalle's principle assure that $F_{0}$ is globally stable.

To perform stability of EE, we shall state and prove the following result.

Theorem 5.3 The disease EE, $F_{*}=\left(S_{*}, L_{*}, A_{*}, C_{*}, R_{*}, V_{*}\right)$ is stable locally asymptotically whenever $R_{0}>1$, while unstable, if $R_{0}<1$.

Proof Clearly the recovered compartment only appears in one of the equation of the proposed model. So it is enough to discuss the reduced system without recovered class. Taking the linearize matrix of the system (1) without recovered compartment around $F_{*}$ and then making use of the elementary row operation we get

$$
J\left(F_{*}\right)=\left(\begin{array}{ccccc}
-q_{1}-\frac{\beta S_{*}}{N}-\frac{\gamma \beta S_{*}}{N} & 0 & -\frac{\beta S_{*}}{N} & -\left(b \xi \eta N+\frac{\gamma \beta S_{*}}{N}\right) & \phi \\
0 & -q_{2} & H_{1} & \gamma H_{1}-b \xi \eta H_{2} & \phi H_{2} \\
0 & 0 & \frac{H_{1}}{q_{2}} \sigma-q_{3} & \frac{\sigma}{q_{2}}\left(H_{1} \gamma-\xi b \eta H_{2}\right) & \frac{\sigma \phi H_{2}}{q_{2}} \\
0 & 0 & 0 & H_{3}-b \xi \eta N H_{4} & \phi H_{4} \\
0 & 0 & 0 & 0 & H_{5}
\end{array}\right),
$$

where

$$
\begin{aligned}
H_{1}= & \frac{q_{1} \beta S_{*}}{A_{*} \beta+d_{0} N+\beta \gamma C_{*}+N \gamma_{3}}, \quad H_{2}=\frac{A_{*} \beta+\beta^{2} \gamma C_{*}}{\beta A_{*}+d_{0} N+\beta \gamma C_{*}+N \gamma_{3}}, \\
H_{3}= & -\frac{\beta \gamma p \gamma_{1} q_{1} S_{*}}{\beta q_{1} \sigma S_{*}-q_{3} q_{2}\left(N d_{0}+A_{*} \beta+\beta \gamma C_{*}+N \gamma_{3}\right)}-q_{4}, \\
H_{4}= & \frac{\beta \sigma \gamma_{1} p\left(A_{*}+C_{*} \gamma\right)}{q_{3} q_{2}\left(N d_{0}+A_{*} \beta+\beta \gamma C_{*}+N \gamma_{3}\right)-\beta \sigma q_{1} S_{*}}, \\
H_{5}= & \frac{\phi \gamma_{3} H_{4}\left(\gamma_{1} p\left(\xi b N^{2} \eta+\beta \gamma S_{*}\right)+\beta S_{*} q_{4}\right)}{\gamma_{1} p\left(N d_{0}+A_{*} \beta+\beta \gamma C_{*}+N \gamma_{3}\right)\left(H_{3}-\eta b \xi H_{4} N\right)} \\
& -\frac{q_{5}\left(N d_{1}+A_{*} \beta+\beta \gamma C_{*}\right)+N \gamma_{3} d_{0}}{N d_{0}+A_{*} \beta+\beta \gamma C_{*}+N \gamma_{3}} .
\end{aligned}
$$


The matrix (17) has the eigenvalues

$$
\begin{aligned}
& \lambda_{1}=-q_{2}, \\
& \lambda_{2}=-\frac{q_{1} N+\beta \gamma C_{*}+\beta A_{*}}{N}, \\
& \lambda_{3}=-\frac{q_{3} q_{2}\left(N d_{0}+A_{*} \beta+\beta \gamma C_{*}+N \gamma_{3}\right)-q_{1} \beta \sigma S_{*}}{q_{2}\left(N d_{0}+A_{*} \beta+\beta \gamma C_{*}+N \gamma_{3}\right)}, \\
& \lambda_{4}=H_{3}-\xi b N H_{4} \eta, \quad \lambda_{5}=H_{5} .
\end{aligned}
$$

Clearly, among the above eigenvalues, two eigenvalues $\lambda_{1}$ and $\lambda_{2}$ are negative, while $\lambda_{i}$, for $i=3,4,5$ are negative, if

$$
\begin{aligned}
& \frac{\gamma \beta \gamma_{1} p S_{*}}{q_{4} q_{2}\left(N d_{0}+A_{*} \beta+\gamma \beta C_{*}+N \gamma_{3}\right)-\beta \gamma q_{4} S_{*}}<1, \\
& \frac{\sigma q_{1} \beta S_{*}}{q_{2} q_{3}\left(d_{0} N+\beta A_{*}+\beta \gamma C_{*}+\gamma_{3} N\right)}<1,
\end{aligned}
$$

which also satisfied. Thus, the eigenvalues of the matrix (17) are negative which proves the conclusion.

Theorem 5.4 The EE $F_{*}$ is globally stable, if $R_{0}>1$, while unstable for $R_{0}<1$.

Proof Taking the Jacobian matrix $J$ and the second additive compound matrix $J^{|2|}$ for only the first three equations of the system (1), we get

$$
J=\left(\begin{array}{ccc}
-a_{11} & 0 & -a_{13} \\
a_{21} & a_{22} & a_{23} \\
0 & \sigma & -a_{33}
\end{array}\right) \quad \text { and } J^{|2|}=\left(\begin{array}{ccc}
-\left(a_{11}+a_{22}\right) & a_{23} & -a_{13} \\
a_{32} & \left.-a_{11}-a_{33}\right) & a_{12} \\
-a_{31} & a_{21} & -\left(a_{22}+a_{33}\right)
\end{array}\right)
$$

Let $P(\chi)=\operatorname{diag}\left\{\frac{S}{L}, \frac{S}{L}, \frac{S}{L}\right\}=P(S, L, A)$, implies $P^{-1}(\chi)=\operatorname{diag}\left\{\frac{L}{S}, \frac{L}{S}, \frac{L}{S}\right\}$, then taking the derivative w.r.t, that is, $P_{f}(\chi)$, we get

$$
P_{f}(\chi)=\operatorname{diag}\left\{\frac{\dot{S}}{S}-\frac{S \dot{L}}{L^{2}}, \frac{\dot{S}}{S}-\frac{S \dot{L}}{L^{2}}, \frac{\dot{S}}{S}-\frac{S \dot{L}}{L^{2}}\right\} .
$$

Now $\operatorname{diag}\left\{\frac{\dot{S}}{S}-\frac{\dot{L}}{L}, \frac{\dot{S}}{S}-\frac{\dot{L}}{L}, \frac{\dot{S}}{S}-\frac{\dot{L}}{L}\right\}=P_{f} P^{-1}$ and $J_{2}^{|2|}=P J_{2}^{|2|} P^{-1}$. We consider $B=P J_{2}^{|2|} P^{-1}+$ $P_{f} P^{-1}$. Alternatively, we can write $B$ as

$$
B=\left(\begin{array}{ll}
B_{11} & B_{12} \\
B_{21} & B_{22}
\end{array}\right)
$$

where

$$
\begin{aligned}
& B_{11}=\frac{\dot{S}}{S}-\frac{\dot{L}}{L}-\frac{A \beta}{N}-\frac{B \beta \gamma}{N}-\gamma_{3}-2 d_{0}-\sigma, \\
& B_{12}=\left[\begin{array}{cc}
\frac{\beta}{N} S & \frac{\beta}{N} S
\end{array}\right], \quad B_{21}=\left[\begin{array}{c}
\sigma \\
0
\end{array}\right],
\end{aligned}
$$




$$
B_{22}=\left[\begin{array}{cc}
\frac{\dot{S}}{S}-\frac{\dot{L}}{L}-A \frac{\beta}{N}-B \gamma \frac{\beta}{N}-\gamma_{1}-2 d_{0}-\sigma & 0 \\
A \frac{\beta}{N}+B \gamma \frac{\beta}{N} & \frac{\dot{S}}{S}-2 d_{0}-\frac{\dot{L}}{L}-\sigma-\gamma_{1}
\end{array}\right]
$$

Assume a vector in $R^{3}$ denoted by $\left(b_{1}, b_{2}, b_{3}\right)$ and $\|\cdot\|$ is its corresponding norm which is defined by

$$
2\left\|b_{1}, b_{2}, b_{3}\right\|=\max \left\{\left\|b_{1}\right\|,\left\|b_{2}\right\|+\left\|b_{3}\right\|\right\} .
$$

Followed [28], we take $\ell(B)$ representing the Lozinski measure with respect to the norm (24), we get

$$
\begin{aligned}
& \ell(B) \leq \sup \left\{g_{i}, i=1,2\right\}=\sup \left\{\left\|B_{12}\right\|+\ell\left(B_{11}\right),\left\|B_{21}\right\|+\ell\left(B_{22}\right)\right\} \\
& g_{i}=\left\|B_{i j}\right\|+\ell\left(B_{i i}\right), \quad \text { for } i \neq j \text { and } i=1,2 .
\end{aligned}
$$

The use of Equation (25) implies

$$
g_{1}=\left\|B_{12}\right\|+\ell\left(B_{11}\right) \text { and } g_{2}=\left\|B_{21}\right\|+\ell\left(B_{22}\right),
$$

where

$$
\begin{aligned}
\ell\left(B_{11}\right) & =\frac{\dot{S}}{S}-\frac{\dot{L}}{L}-A \frac{\beta}{N}-B \frac{\beta}{N} \gamma-\gamma_{3}-2 d_{0}-\sigma, \\
\ell\left(B_{22}\right) & =\max \left\{\frac{\dot{S}}{S}-\frac{\dot{L}}{L}-\gamma_{3}-\gamma_{1}-2 d_{0}, \frac{\dot{S}}{S}-2 d_{0}-\frac{\dot{L}}{L}-\sigma-\gamma_{1}\right\} \\
& =\frac{\dot{S}}{S}-2 d_{0}-\frac{\dot{L}}{L}-\min \left\{\gamma_{3}, \sigma\right\}-\gamma_{1}, \\
\left\|B_{12}\right\| & =\frac{\beta}{N} S, \quad\left\|B_{21}\right\|=\max \{\sigma, 0\}=\sigma .
\end{aligned}
$$

Thus, $g_{1}$ and $g_{2}$ becomes, $g_{1} \leq \frac{\dot{S}}{S}-\sigma-\gamma_{3}-2 d_{0}$ and $g_{2} \leq \frac{\dot{S}}{S}-\gamma_{1}-2 d_{0}+\sigma-\min \left\{\gamma_{3}, \sigma\right\}$, which implies that $\ell(B) \leq\left\{\frac{\dot{S}}{S}+\sigma-\min \left\{\gamma_{3}, \sigma\right\}-2 d_{0}\right\}$. Hence, $-2 \mu_{0}+\ell(B) \leq \frac{\dot{S}}{S}$. Integrating $\ell(B)$ in $[0, t]$ and then by taking $\lim _{t \rightarrow \infty}$, yields

$$
\lim _{t \rightarrow \infty} \sup \sup \frac{1}{t} \int_{0}^{t} \ell(B) d t<-2 \mu_{0} .
$$

Finally, we obtain

$$
\bar{q}=\lim _{t \rightarrow \infty} \sup \sup \frac{1}{t} \int_{0}^{t} \ell(B) d t<0 .
$$

Hence, the system which contains only first three equations of the system (1) is stable globally asymptotically around $\left(S_{*}, L_{*}, A_{*}\right)$. Also for the remaining taking the limiting system whose solution yields $C(t)$ and $V(t)$ which approaches to $C_{*}$ and $V_{*}$, respectively as $t \rightarrow \infty$.

\section{Numerical Simulation}

We present the numerical solutions for the verification of theoretical findings of the proposed model (1). We use Runge-Kutta of fourth order method and different values of the parameter 
for this purpose of the model (1). The parameters used in the numerical simulation are taken with biologically feasible way given in Table 2 .

Taking $0-200$ units is the interval of time, while the size of population for $S(t), L(t), A(t)$, $C(t), R(t)$ and $V(t)$ are given in Table 2 .

Table 2 Parameter values, weight constants and initial size of compartmental population used in numerical simulation

\begin{tabular}{|c|c|c|c|}
\hline Parameter & parameter description & value & Source \\
\hline$b$ & new born rate & 0.0121 & {$[21]$} \\
\hline$\xi$ & $\begin{array}{l}\text { new born with unsuccessful vaccina- } \\
\text { tion }\end{array}$ & 0.0500 & {$[21]$} \\
\hline$\eta$ & ratio of maternally infected & 0.0110 & {$[21]$} \\
\hline$\phi$ & induced rate of waning vaccine & 0.1000 & {$[21]$} \\
\hline$\sigma$ & rate of latency to acute & 0.0012 & {$[21]$} \\
\hline$\beta$ & hepatitis B transmission rate & 0.0950 & {$[21]$} \\
\hline$\gamma_{1}$ & rate of acute to chronic & 0.3300 & {$[21]$} \\
\hline$\gamma_{2}$ & rate of chronic to immune & 0.0090 & {$[21]$} \\
\hline$\gamma_{3}$ & vaccination rate & 0.0200 & {$[21]$} \\
\hline$d_{0}$ & natural mortality rate & 0.0121 & {$[21]$} \\
\hline$d_{1}$ & disease-related mortality rate & 0.0026 & {$[21]$} \\
\hline$p$ & $\begin{array}{l}\text { probability of fails individual, who } \\
\text { recovers in acute stage }\end{array}$ & 0.8000 & $\begin{array}{l}\text { Assumed with the } \\
\text { help of }[21]\end{array}$ \\
\hline$A_{1}$ & weight constant for $S(t)$ & 1000.0 & {$[32]$} \\
\hline$A_{2}$ & weight constant for $L(t)$ & 0.6000 & Fitted \\
\hline$A_{3}$ & weight constant for $A(t)$ & 10.000 & Fitted \\
\hline$A_{4}$ & weight constant for $C(t)$ & 0.9000 & $\begin{array}{l}\text { Assumed with the } \\
\text { help of [32] }\end{array}$ \\
\hline$B_{1}$ & weight constant for $u_{1}(t)$ & 0.4400 & Assumed \\
\hline$B_{2}$ & weight constant for $u_{2}(t)$ & 0.2000 & Fitted \\
\hline$B_{3}$ & weight constant for $u_{3}(t)$ & 1000.0 & Assumed \\
\hline$S(0)$ & susceptible & 1000.0 & {$[21]$} \\
\hline$L(0)$ & latent & 400.00 & {$[21]$} \\
\hline$A(0)$ & acute & 300.00 & {$[21]$} \\
\hline$C(0)$ & chronic & 200.00 & {$[21]$} \\
\hline$R(0)$ & recovered & 100.00 & {$[21]$} \\
\hline$V(0)$ & vaccinated & 100.00 & {$[21]$} \\
\hline
\end{tabular}




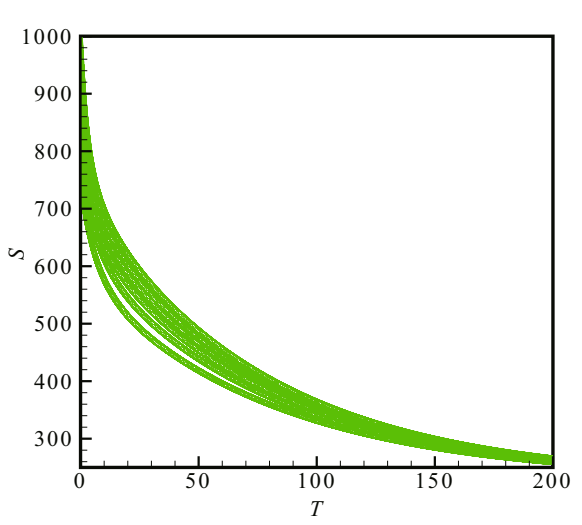

(a) Susceptible individuals

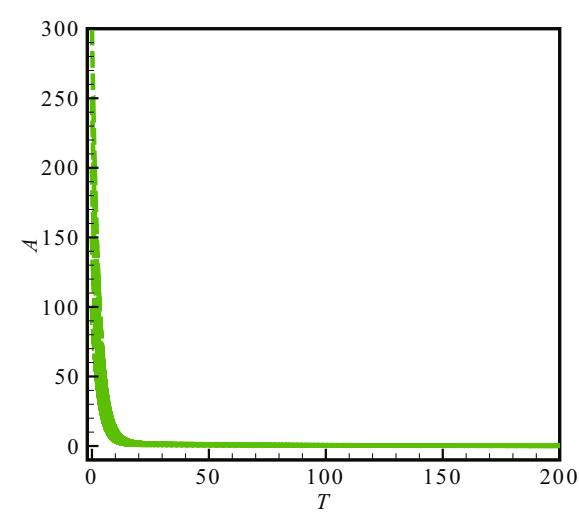

(c) Acutely infected hepatitis B individuals

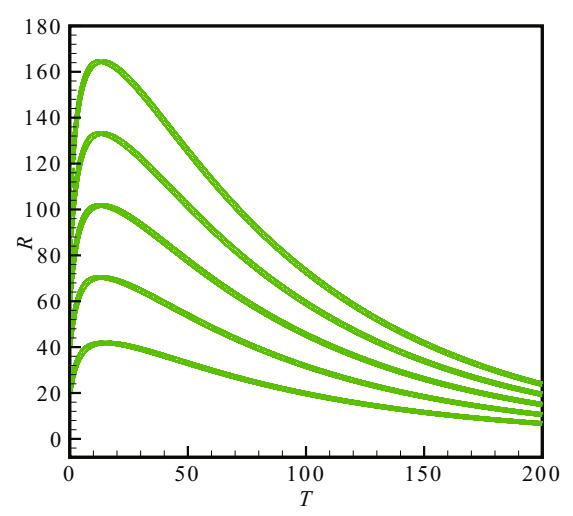

(e) Recovered individuals

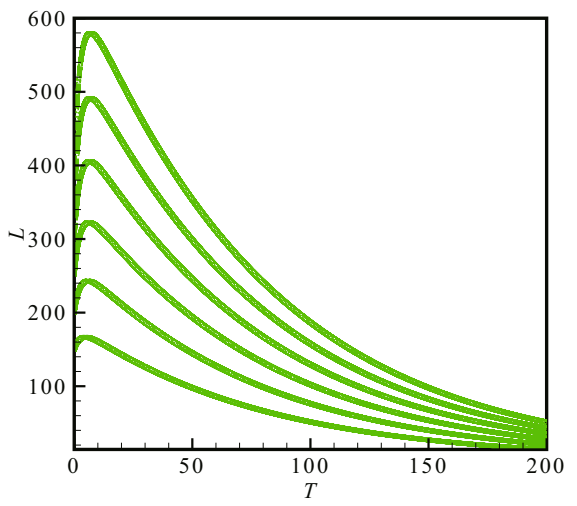

(b) Latent individuals

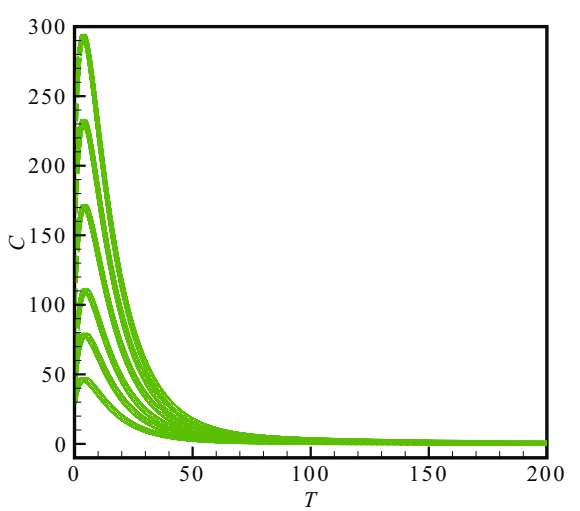

(d) Chronically infected hepatitis B individuals

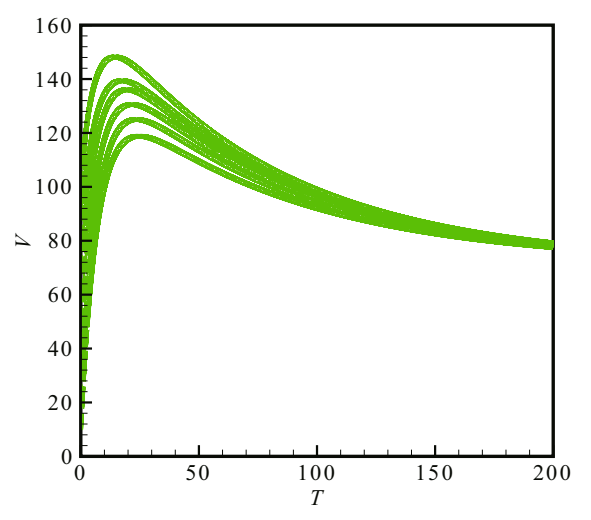

(f) Vaccinated individuals

Figure 1 Stability curves of (1) with different initial size of population 
The simulation part of the study shows that the susceptible population will usually exist. Similarly there is also always vaccinated individuals, but latent, acutely infected, chronic carrier and recovered vanishes, see Figure 1 . The trajectories of $S(t), L(t), A(t)$, carriers $B(t), R(t)$ and $V(t)$ go to the equilibrium points as shown in Figures $(1(\mathrm{a}))-(1(\mathrm{f}))$. This proves the stability of the model (1).

\section{Local Sensitivity}

We perform the local sensitivity of the threshold quantity $R_{0}$ to define the relative change of the parameters to the infection transmission. It decides the sturdiness of model prediction to the values of the parameter. Commonly during data collection, the uncertainties as well as the estimation of the parameters value considerably affect the threshold quantity.

Definition 7.1 The normalized sensitivity index of $R_{0}$ with respect to $\Phi$ is defined by

$$
S_{\Phi}=\frac{\Phi}{R_{0}} \frac{\partial R_{0}}{\partial \Phi} .
$$

We accomplish the sensitivity indices of $R_{0}$ to the model parameters, which allow to quantify the comparative variation in $R_{0}$ with the variation in a value of the parameter. By the use of these indices, we are able to point out the most risky parameters that extremely affect the threshold quantity $R_{0}$.

Table 3 represents that $\sigma, \beta, \eta, \xi$ and $\phi$ have a direct relation with $R_{0}$. Which mean that increase or decrease in these values by $10 \%$ will increase or decrease $R_{0}$ respectively by $10 \%, 9.0 \%, 5.46 \%, 5.46 \%$ and $8.13 \%$, see for detail Figure 2. However, on the other side parameters $\gamma_{1}$ and $\gamma_{3}$ have an inverse relation with $R_{0}$, which illustrates, that increase in its values by $10 \%$ will reduce $R_{0}$ by $7.38 \%$ and $8.24 \%$, respectively as presented in Figure 2 .

Table 3 The sensitive indices of $R_{0}$

\begin{tabular}{lcc}
\hline Parameter & Sensitivity index & value \\
\hline Hepatitis B transmission rate $(\beta)$ & $S_{\beta}$ & +1.0000 \\
Moving rate from L to A $(\sigma)$ & $S_{\sigma}$ & +0.9097 \\
Recovery rate in A $\left(\gamma_{1}\right)$ & $S_{\gamma_{1}}$ & -0.8247 \\
Recovery rate in C $\left(\gamma_{2}\right)$ & $S_{\gamma_{2}}$ & -0.7385 \\
Vaccination $\left(\gamma_{3}\right)$ & $S_{\gamma_{3}}$ & -0.1514 \\
Proportion of vertically infected & $S_{\eta}$ & +0.5460 \\
population $(\eta)$ & & \\
$\begin{array}{l}\text { Birth without successful vaccination } \\
(\xi)\end{array}$ & $S_{\xi}$ & +0.5460 \\
waning vaccine induced immunity & $S_{\phi}$ & +0.8135 \\
rate $(\phi)$ & & \\
\hline
\end{tabular}




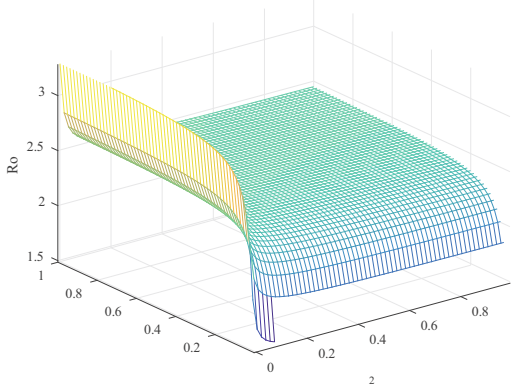

(a) $\gamma_{2}$ and $\sigma$ verses $R_{0}$

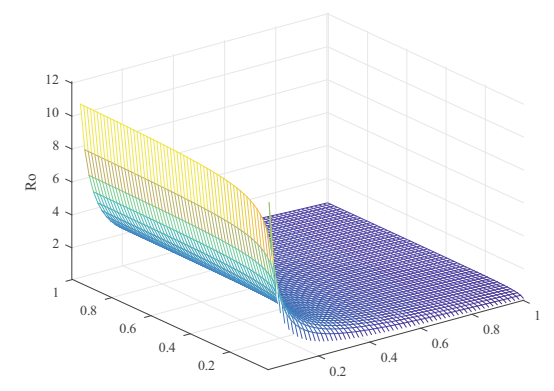

(c) $\phi$ and $\gamma_{1}$ verses $R_{0}$

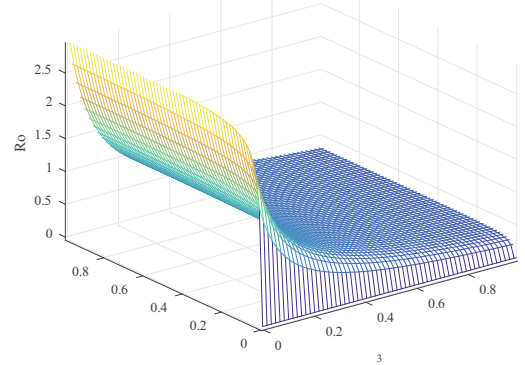

(b) $\gamma_{3}$ and $\sigma$ verses $R_{0}$

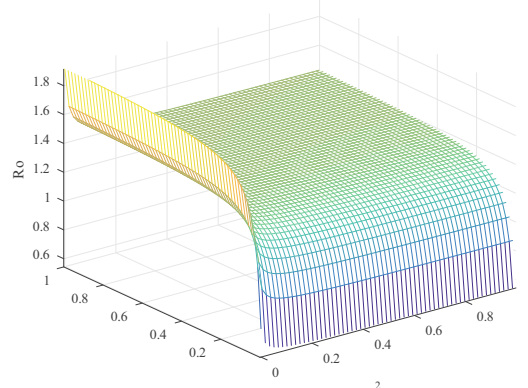

(d) The scatter plot of residuals

Figure 2 The plots represent the sensitivity of $R_{0}$ w.r.t parameters

In order to eliminate the HBV infection, we need more attention to reduce the transmission rate $\beta$. Because this parameter has got the maximum index 1 , which demonstrates that the reduction of this parameter by ten percent reduce the value of the $R_{0}$ by ten percent. Among these parameters, $\gamma_{1}$ got the second highest index, which is -0.8247 , which means that if we increase the value of $\gamma_{1}$ by 10 percent, the value of $R_{0}$ will be decrease by 8.24 percent. The sensitivity index of the parameters $\eta, \sigma, \phi$ and $\xi$ is 2.8152 collectively. So 10 percent decrease in these parameters causes 28.152 percent decreases in the value of $R_{0}$. Similarly 1.5623 is the sensitivity index of $\gamma_{2}$ and $\gamma_{1}$, therefore if we increase the value of these parameters by $10 \%$ will decrease $R_{0}$ by $15.632 \%$. Hence, by using this analysis, it is handy to formulate control strategy for the eradication of the HBV infection.

\section{Formulation of the Optimal Control Problem}

Optimal control theory and calculus of variations are the two techniques which can be used for minimization/maximization problems and many researchers used control theory to make a control strategy for eliminating hepatitis B see, for example, [6, 9, 11, 29-32] and the references cited therein. Control theory applied to HBV infection and related work have been regorously investigated in [33-38]. Based on these studies, we shall focus on optimizing the spreading of infection rate $\beta$, whose sensitivity index is 1 . The decreases in this rate by $10 \%$ will reduce the value of $R_{0}$ by 10 percent. The second recovery rate $\gamma_{1}$ of acutely hepatitis $\mathrm{B}$ infected 
individuals has maximum sensitivity index -0.8247 , which has a inverse relation with $R_{0}$. So an increase in the recovery rate $\gamma_{1}$ say by $10 \%$ reduce $R_{0}$ value by 8.247 percent. The index of recovery rate $\gamma_{2}$ of chronic carriers is -0.7385 . The increase of the recovery rate $\gamma_{2}$ by say by $10 \%$ will also decrease the value of $R_{0}$ by 7.385 percent. Similarly the collectively index of $\eta, \sigma, \phi$ and $\xi$ is 2.8152. If we decrease these parameters say by $10 \%$, will decrease $R_{0}$ by $28.152 \%$. The parameter $\gamma_{3}$ got the sensitivity index -0.1514 , i.e., increase of $\gamma_{3}$ by $10 \%$ will effect reduction in the value of $R_{0}$ by 1.514 percent.

We coupled all the effect of these parameters parameters $\beta, \xi, \eta, \sigma, \gamma_{1}, \gamma_{2}, \gamma_{3}$ and $\phi$. Growth or decay in the above mentioned parameters can produce the change in the rest parameters. Therefore, on the basis of the above sensitivity indexes, we formulate optimal strategies by introducing the following time dependent control variables.

i) The control measure $u_{1}(t)$ represents the isolation of non-infected and infected populations. The role of this control is to reduce the hepatitis $\mathrm{B}$ transmission rate $\beta$.

ii) The control variable $u_{2}(t)$ represents the control variable treatment of hepatitis B infected individuals. Through this control variable, we want to reduce the number of infected individuals.

iii) $u_{3}(t)$ represents the control measure vaccination of hepatitis B, i.e., through this control variable, we want to maximize vaccinated population.

Thus, the use of these three variables our control problem become the modified version of the system (1) given by

$$
\begin{aligned}
\frac{d S(t)}{d t}= & \xi b(1-\eta C(t)) N+\phi V(t)-\frac{\beta S(t) A(t)}{N}\left(1-u_{1}(t)\right)-\frac{\gamma \beta S(t) C(t)}{N}\left(1-u_{1}(t)\right) \\
& -\left(\gamma_{3}+d_{0}+u_{3}(t)\right) S(t), \quad S(0)>0 \\
\frac{d L(t)}{d t}= & \frac{\beta}{N} A(t) S(t)\left(1-u_{1}(t)\right)+\frac{\beta}{N} \gamma C(t) S(t)\left(1-u_{1}(t)\right)-\left(d_{0}+\sigma\right) L(t) \\
& -\left(u_{2}(t)+u_{3}(t)\right) L(t), \quad L(0) \geq 0, \\
\frac{d A(t)}{d t}= & \sigma L(t)-\left(d_{0}+\gamma_{1}+u_{2}(t)+u_{3}(t)\right) A(t), \quad A(0) \geq 0 \\
\frac{d C(t)}{d t}= & \xi b \eta C(t) N+\gamma_{1} p A(t)-\left(d_{1}+\gamma_{2}+u_{2}(t)+d_{0}+u_{3}(t)\right) C(t), \quad C(0) \geq 0 \\
\frac{d R(t)}{d t}= & \gamma_{2} C(t)+(1-p) \gamma_{1} A(t)-d_{0} R(t)+(L(t)+A(t)+C(t)) u_{2}(t), \quad R(0) \geq 0 \\
\frac{d V(t)}{d t}= & (1-\xi) N b+\gamma_{3} S(t)-\left(d_{0}+\phi\right) V(t)+(S(t)+L(t)) u_{3}(t) \\
& +(A(t)+C(t)) u_{3}(t), \quad V(0) \geq 0 .
\end{aligned}
$$

The goal of the control strategies is to reduce the acute, chronic and latent population; and the associated cost of $u_{1}(t), u_{2}(t)$ and $u_{3}(t)$. We will assume that the control variables are Lebesgue measurable and bounded. Thus, the objective functional becomes

$$
\begin{aligned}
J\left(u_{1}, u_{2}, u_{3}\right)=\int_{0}^{t_{f}} & {\left[A_{1} S(t)+A_{2} L(t)+A_{3} A(t)+A_{4} C(t)\right.} \\
& \left.+\frac{1}{2}\left(B_{1} u_{1}^{2}(t)+B_{2} u_{2}^{2}(t)+B_{3} u_{3}^{2}(t)\right)\right] d t,
\end{aligned}
$$


subject to the proposed model (30). In the objective functional (31), $A_{1}, A_{2}, A_{3}$ and $A_{4}$ represent the weight constants of the susceptible individuals, latent individuals, acute and carrier population, respectively. The constants $B_{1}, B_{2}$ and $B_{3}$ represent the weight constants associated to the controls. More preciously these constants represent the weight constants for isolation, treatment and vaccination, respectively. The terms $\frac{1}{2} B_{1} u_{1}^{2}(t), \frac{1}{2} B_{2} u_{2}^{2}(t)$ and $\frac{1}{2} B_{3} u_{1}^{3}(t)$ represents cost of disease intervention. The cost related with strategy $u_{1}(t)$ produces due to the cost of isolation of infected and non-infected individuals. The cost associated to the control strategy $u_{2}(t)$ produces due to medication of infected individuals. The regarding strategy $u_{3}(t)$ is the cost of vaccination of hepatitis $\mathrm{B}$.

Our goal here is to decrease the size of susceptible, latent, acute and chronic population, and to increase the number of recovered individuals and vaccinated population by using the three time dependent control variables $u_{i}(t), i=1,2,3$. We find the control function, such that

$$
J\left(u_{1}^{*}, u_{2}^{*}, u_{3}^{*}\right)=\min \left\{J\left(u_{1}, u_{2}, u_{3}\right), u_{1}, u_{2}, u_{3} \in U\right\}
$$

subject to the proposed model (30), where the control set is defined as,

$$
U=\left\{\left(u_{1}, u_{2}, u_{3}\right) \mid u_{i}(t) \text { are Lebesgue measurable on }[0,1], 1 \geq u_{i}(t) \geq 0, i=1,2,3\right\} .
$$

Before moving further, we need to show that such control measures exist.

\subsection{Existence of Solution}

In this subsection, we will show that a solution of the control problem (30) exist. For nonnegative subsidiary conditions and bounded Lebesgue measurable controls functions, there exist positive bounded solution to the state system ${ }^{[39]}$. To show the existence of solution of the proposed control problem (30), we investigate the following result.

Theorem 8.1 There exist a set of control measures $u^{*}=\left(u_{1}^{*}, u_{2}^{*}, u_{3}^{*}\right) \in U$, such that

$$
J\left(u_{1}^{*}, u_{2}^{*}, u_{3}^{*}\right)=\min J\left(u_{1}, u_{2}, u_{3}\right),
$$

subject to the control system (30).

Proof Clearly the following holds

i) The control measures $u_{i}(t)$ for $i=1,2,3$ and the state variables $(S, L, A, C, R, V)$ are nonnegative.

ii) The control set $U$ is convex and closed.

iii) Boundedness of the optimal system assures the compactness.

iv) The integrand $A_{1} S(t)+A_{2} L(t)+A_{3} A(t)+A_{4} C(t)+\frac{1}{2}\left(B_{1} u_{1}^{2}+B_{2} 2 u_{2}^{2}+B_{3} u_{3}^{2}\right)$ in (31) is convex on $U$, which completes the proof. 


\subsection{Optimality Condition}

To characterize the optimal solution for (30), let us define the Lagrangian and the Hamiltonian associated with our optimal problem. Thus, the Lagrangian is given by

$$
\begin{aligned}
L\left(S, L, A, C, u_{1}, u_{2}, u_{3}\right)= & A_{1} S(t)+A_{2} L(t)+A_{3} A(t)+A_{4} C(t) \\
& +\frac{1}{2}\left(B_{1} u_{1}^{2}(t)+B_{2} u_{2}^{2}(t)+B_{3} u_{2}^{3}(t)\right) .
\end{aligned}
$$

For the purpose of minimal value of the Lagrangian, we define $H$ (Hamiltonian) for (30) as

$$
H(x, u, \lambda)=L(x, u)+\lambda \cdot F(x, u),
$$

where

$$
\begin{aligned}
x=(S, L, A, C), \quad u=\left(u_{1}, u_{2}, u_{3}\right), \quad \lambda=\left(\lambda_{1}, \lambda_{2}, \cdots, \lambda_{6}\right), \\
F_{1}(x, u)=\phi V(t)+b \xi N(1-\eta C(t))-\left(\frac{\beta}{N} S(t) A(t)+\frac{\beta}{N} \gamma S(t) C(t)\right)\left(1-u_{1}(t)\right) \\
\quad-\left(\gamma_{3}+d_{0}+u_{3}(t)\right) S(t), \\
F_{2}(x, u)=\left(\frac{\beta}{N} A(t) S(t)+\frac{\beta \gamma S(t) C(t)}{N}\right)\left(1-u_{1}(t)\right)-\left(d_{0}+\sigma\right) L(t) \\
\quad-\left(u_{2}(t)+u_{3}(t)\right) L(t), \\
F_{3}(x, u)=\sigma L(t)-\left(\gamma_{1}+d_{0}+u_{2}(t)+u_{3}(t)\right) A(t), \\
F_{4}(x, u)=\xi b \eta C(t) N+\gamma_{1} p A(t)-\left(d_{1}+\gamma_{2}+d_{0}+u_{2}(t)+u_{3}(t)\right) C(t), \\
F_{5}(x, u)=\gamma_{2} C(t)+(1-p) \gamma_{1} A(t)-d_{0} R(t)+(L(t)+A(t)+C(t)) u_{2}(t), \\
F_{6}(x, u)=b N(1-\xi)+\gamma_{3} S(t)-\left(d_{0}+\phi\right) V(t)+(S(t)+L(t)) u_{3}(t) \\
\quad+(A(t)+C(t)) u_{3}(t),
\end{aligned}
$$

and $F(x, u)=\left(F_{1}, F_{2}, \cdots, F_{6}\right)(x, u)$. For the optimal solution to the developed control problem, we will prefer to use Pontryagin'n Maximum Principle ${ }^{[28]}$ : If $\left(x^{*}, u^{*}\right)$ is an optimal solution to (30) with $u^{*}$ being essentially bounded, then there exists a non-trivial vector function $\lambda$, such that the Hamiltonian system

$$
\left\{\begin{array}{c}
\frac{d x^{*}(t)}{d t}=\frac{\partial H}{\partial \lambda}\left(x^{*}(t), u^{*}(t), \lambda(t)\right), \\
\frac{d \lambda(t)}{d t}=-\frac{\partial H}{\partial x}\left(x^{*}(t), u^{*}(t), \lambda(t)\right), \\
0=\frac{\partial H}{\partial u}\left(x^{*}(t), u^{*}(t), \lambda(t)\right),
\end{array}\right.
$$

the maximality condition

$$
H\left(x^{*}(t), u^{*}(t), \lambda(t)\right)=\max _{u_{1}, u_{2}, u_{3} \in[0,1]} H\left(x^{*}(t), u_{1}, u_{2}, u_{3}, \lambda(t)\right) ;
$$

and the transversality condition

$$
\lambda\left(t_{f}\right)=0
$$

hold. 
Theorem 8.2 Let $S^{*}, L^{*}, A^{*}, C^{*}, R^{*}$ and $V^{*}$ be optimal states solutions associated to essentially bounded optimal controls $\left(u_{1}^{*}, u_{2}^{*}, u_{3}^{*}\right)$ for the optimal control problem (30). Then there exist adjoint variables $\lambda_{i}(t), i=1,2, \cdots, 6$, satisfying

$$
\begin{aligned}
\frac{d \lambda_{1}(t)}{d t}= & \left(\lambda_{1}(t)-\lambda_{2}(t)\right)\left(\frac{\beta A^{*}+\gamma \beta C^{*}}{N}\left(1-u_{1}(t)\right)\right)+\lambda_{1}(t)\left(d_{0}+\gamma_{3}+u_{3}(t)\right)-A_{1} \\
& -\lambda_{6}(t)\left(\gamma_{3}+u_{3}(t)\right), \\
\frac{d \lambda_{2}(t)}{d t}= & \lambda_{2}(t)\left(\sigma+d_{0}+u_{2}(t)+u_{3}(t)\right)-\sigma \lambda_{3}(t)-\lambda_{5}(t) u_{2}(t)-\lambda_{6}(t) u_{3}(t)-A_{2}, \\
\frac{d \lambda_{3}(t)}{d t}= & \left(\lambda_{1}(t)-\lambda_{2}(t)\right)\left(\frac{\beta S^{*}}{N}\left(1-u_{1}(t)\right)\right)+\lambda_{3}(t)\left(d_{0}+\gamma_{1}+u_{2}(t)+u_{3}(t)\right) \\
& -\lambda_{4}(t) p \gamma_{1}-\lambda_{5}(t)\left((1-p) \gamma_{1}+u_{2}(t)\right)-\lambda_{6}(t) u_{3}(t)-A_{3}, \\
\frac{d \lambda_{4}(t)}{d t}= & -A_{4}+\left(\lambda_{1}(t)-\lambda_{2}(t)\right)\left(\frac{\gamma \beta S^{*}}{N}\left(1-u_{1}(t)\right)\right)+\lambda_{1}(t) b \xi \eta N \\
& -\lambda_{4}(t)\left(b \xi \eta N-\left(d_{0}+d_{1}+\gamma_{2}+u_{2}(t)+u_{3}(t)\right)\right)-\lambda_{5} u_{2}(t)-\lambda_{6}(t) u_{3}(t), \\
\frac{d \lambda_{5}(t)}{d t}= & d_{0} \lambda_{5}(t), \\
\frac{d \lambda_{6}(t)}{d t}= & \lambda_{1}(t) \phi+\left(d_{0}+\phi\right) \lambda_{6}(t),
\end{aligned}
$$

with transversality conditions

$$
\lambda_{i}\left(t_{f}\right)=0 \text { for } i=1,2, \cdots, 6
$$

Furthermore, the optimal controls variable $u_{1}^{*}(t), u_{2}^{*}(t)$ and $u_{3}^{*}(t)$ are given by

$$
\begin{aligned}
& u_{1}^{*}=\max \left\{\min \left\{\frac{\left(\beta S^{*} A^{*}+\gamma \beta S^{*} C^{*}\right)\left(\lambda_{2}(t)-\lambda_{1}(t)\right)}{N B_{1}}, 1\right\}, 0\right\}, \\
& u_{2}^{*}=\max \left\{\min \left\{\frac{\left(\lambda_{2}(t)-\lambda_{5}(t)\right) L^{*}+\left(\lambda_{3}(t)-\lambda_{5}(t)\right) A^{*}+\left(\lambda_{4}(t)-\lambda_{5}(t)\right) C^{*}}{B_{2}}, 1\right\}, 0\right\}, \\
& u_{3}^{*}=\max \left\{\min \left\{\frac{\left(\lambda_{1}(t) S^{*}+\lambda_{2}(t) L^{*}+\lambda_{3}(t) A^{*}+\lambda_{4}(t) C^{*}-\lambda_{6}(t)\right)\left(S^{*}+L^{*}+A^{*}+C^{*}\right)}{B_{3}}, 1\right\}, 0\right\} .
\end{aligned}
$$

Proof System (40) is direct comes from applying adjoint equation of the Pontryagin Maximum Principle to our problem, that is, from the 2nd equation in (37) with the Hamiltonian $H$ defined by (34)-(36), while conditions (41) is a direct consequence of the transversality condition (39). To obtain $u_{1}^{*}, u_{2}^{*}$ and $u_{3}^{*}$, we differentiate the Hamiltonian with respect to control measures, then we solve the system $\frac{\partial H}{\partial u_{1}}=0, \frac{\partial H}{\partial u_{2}}=0$ and $\frac{\partial H}{\partial u_{3}}=0$ (necessary optimality conditions for the finite dimension optimization problem on the right-hand side of (38), when one restrict himself to the interior $(0,1) \times(0,1)$ of the optimization region). Lastly, by using the maximality condition (38), we obtain the optimal control measures $u_{1}^{*}, u_{2}^{*}$ and $u_{3}^{*}$, which completes the proof.

We found the optimal state and control variables by solving numerically the optimality system, which contains the state system (30) and the adjoint system (40), boundary conditions (41) 
and (41), together with the characterization of the optimal values of the controls $\left(u_{1}^{*}, u_{2}^{*}, u_{3}^{*}\right)$. In addition, the Hessian matrix of the Hamiltonian with respect to $u_{1}, u_{2}$ and $u_{3}$ should be positive semidefinite, describing the fact that we are considering a minimization problem.

\section{Numerical Analysis of the Optimal Problem}

To simulate the control problem, we intend to use Runge-Kutta procedure of order four. More precisely, we wish to solve the state system (30) by using the Runge-Kutta scheme of order four with initial conditions (41) forward in time [0,100], and subsequently solving the adjoint system (40) by the backward Runge-Kutta of order four in the same interval of time with the help of the transversality conditions (41) and the solution of the state system. For simulation purposes, the following parameter values were chosen are given in Table 2. The results presented in Figures 3-5 were obtained. Figure 3 represents the graphs of susceptible population, latent population, acute hepatitis B infected population and chronically infected hepatitis B population with and without (optimal) control. The simulations illustrate clearly our objective in applying the controls: To reduce the number of susceptible, latent, acute and chronically infected with hepatitis B population, and to increase the number of recovered individuals and vaccinated population. Figure 3(a) represents the graph of susceptible individuals with and without (optimal) control. Similarly, Figures 3(b)-(d) represents the graph of latent, acute, chronically infected individuals with and without (optimal) control, while Figure 3 represents the graphs of recovered and vaccinated individuals with and without (optimal) control. Furthermore, Figure 3 represents the dynamic of the optimal control variables. Clearly, the difference between the two cases is visible.

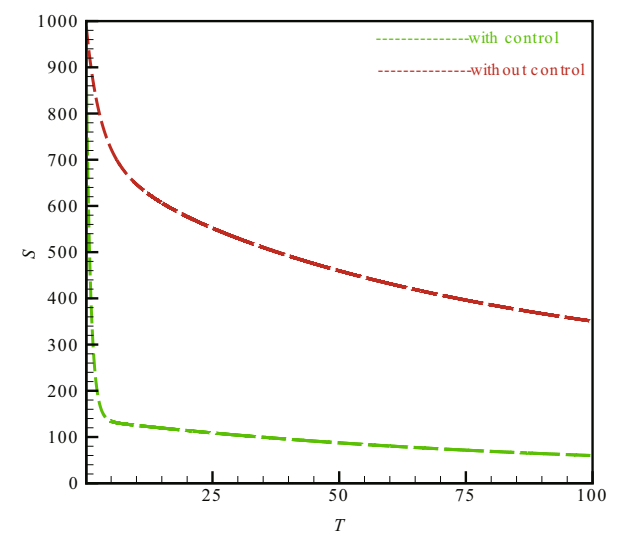

(a) Susceptible population

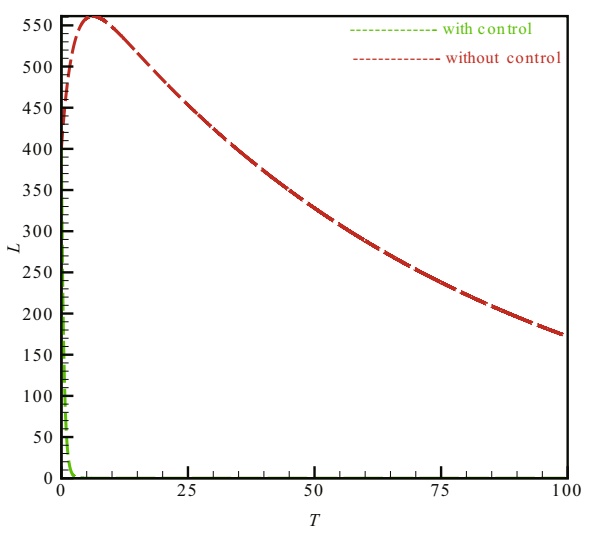

(b) Latent population 


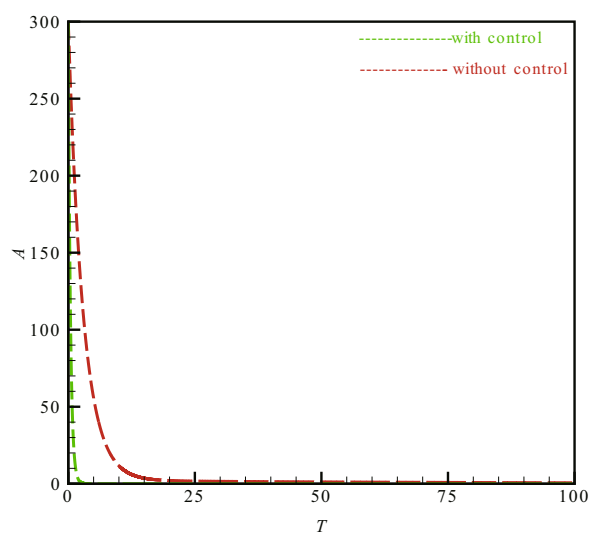

(c) Acute population

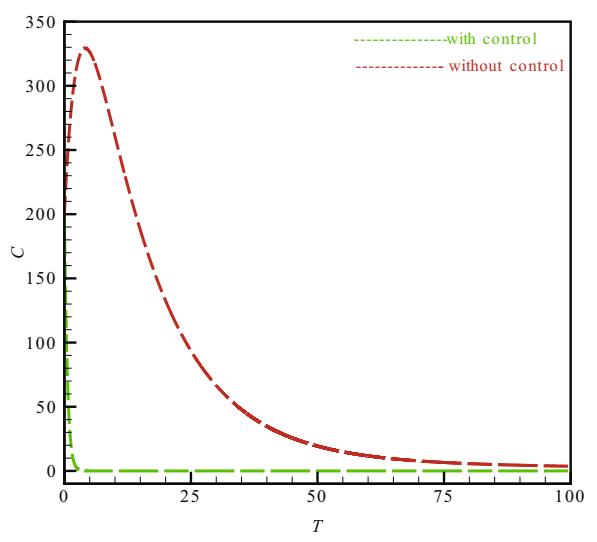

(d) Chronic population

Figure 3 The time dynamics of the compartmental population model (1) with and without control

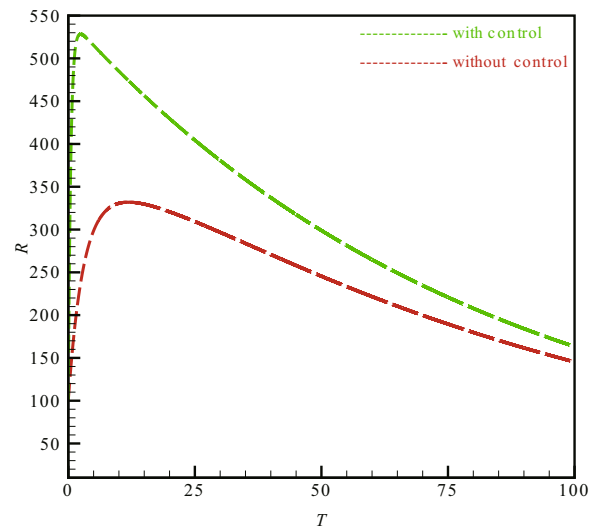

(a) Recovered population

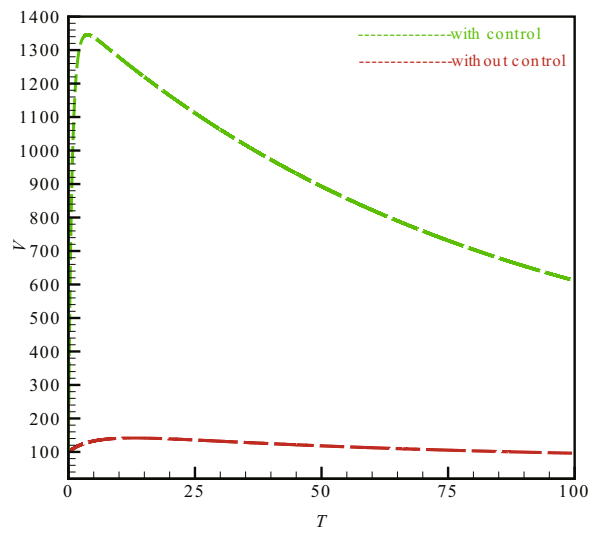

(b) Vaccinated population

Figure 4 The time dynamics of the recovered and vaccinated population with and without control 


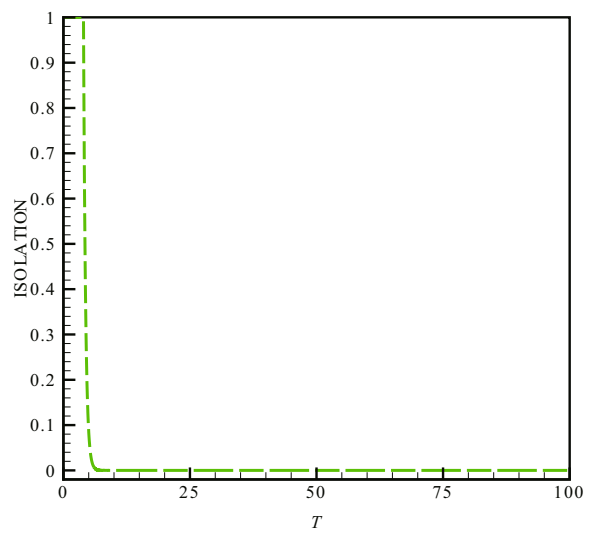

(a) Optimal control variable isolation $u_{1}(t)$

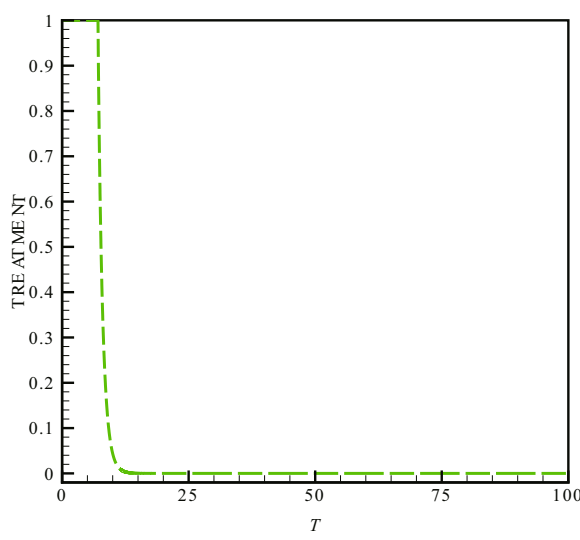

(b) Optimal control variable treatment $u_{2}(t)$

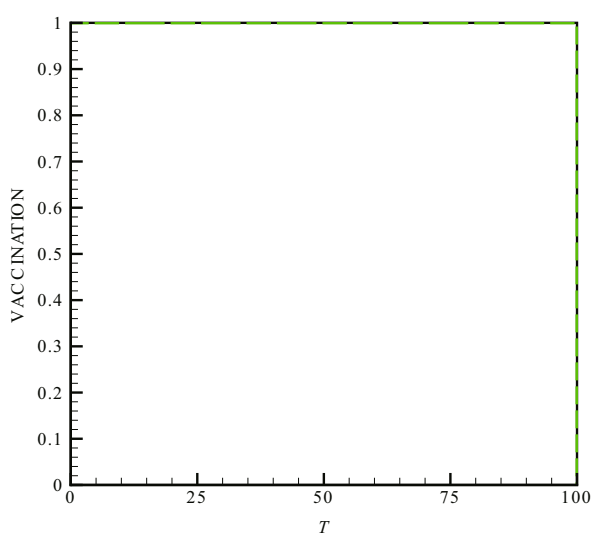

(c) Optimal control variable vaccination $u_{3}(t)$

Figure 5 The time dynamics of the optimal control variables

\section{Discussion and Conclusion}

In this section, the discussion and concluding remarks are presented. First, the discussion of the numerical simulation is presented. The control variables $u_{1}(t), u_{2}(t)$ and $u_{3}(t)$ reduces the transmission rate $\beta$, the moving rate $\sigma$ of latent individuals to acute class, the proportion of parentally infected individuals $\eta$, the birth rate without successful vaccination $\xi$ and the waning vaccine induced immunity rate $\phi$. Which causes decrease in the transmission and consequently decrease the susceptible individual $S(t)$, the latent individual $L(t)$, the acute hepatitis B individuals $A(t)$ and the chronic carrier individuals $C(t)$ as shown in Figures 3 (a)-(d). While causes increase in the non-infected individuals, which consequently increase the recovered individuals $R(t)$ and $V(t)$ as shown in Figures 4 (a)-(b). The graph of susceptible population approaches to a small number due to optimal control $u_{1}(t)$ and $u_{2}(t)$ as shown 
in Figure 3 (a). Similarly the graph of latent population, acute infected and chronic carrier population approaches to a small number due to optimal control shown in Figures 3 (b)-(d). While Figures 4 (a)-(b) shows that the recovered and vaccinated individuals are approaches to a large number in case of with optimal control.

In this work, we studied hepatitis B epidemic model with nonlinear incidence. We studied different mathematical properties of boundedness and positivity to show the biological feasibility. We found reproduction number and discussed the sensitivity analysis. We also used the optimization theory and developed a control strategy on the basis of normalized sensitive index to eliminate hepatitis B from the population. By using the normalized sensitivity index, we investigated the most sensitive parameters and then developed the control strategy. For this purpose, we used three time-dependent control measures, the isolation of non-infected and infected individuals, $u_{1}(t)$, the treatment, $u_{2}(t)$ and the vaccination, $u_{3}(t)$. Once, we formulated the optimal control problem, then proved the existence and characterize the optimality condition using the Pontryaginn Maximum Principle. Finally, the numerical simulation of the optimal problem are presented to support the analytical work.

\section{References}

[1] Zou L, Ruan S, and Zhang W, On the sexual transmission dynamics of hepatitis B virus in China, Journal of Theoretical Biology, 2015, 369: 1-12.

[2] Haq F, Shah K, Khan A, et al., Numerical solution of fractional order epidemic model of a vector born disease by Laplace Adomian decomposition method, Punjab University Journal of Mathematics, 2017, 49(2): 13-22.

[3] Haq F, Shah A, Rahman G U, et al., Numerical solution of fractional order smoking model via Laplace Adomian decomposition method, Alexandria Engineering Journal, 2018, 57(2): 10611069.

[4] Pang L, Ruan S, Liu S, et al., Transmission dynamics and optimal control of measles epidemics, Applied Mathematics and Computation, 2015, 256: 131-147.

[5] Khan A and Zaman G, Asymptotic behavior of an age structure SIRS endemic model, Applied and Computational Mathematics, 2018, 17(2): 185-204.

[6] Zaman G, Kang Y H, and Jung I H, Stability analysis and optimal vaccination of an SIR epidemic model, BioSystems, 2008, 93: 240-249.

[7] Zeb A, Zaman G, and Momani S, Square-root dynamics of a giving up smoking model, Applied Mathematical Modelling, 2013, 37: 5326-5334.

[8] Rahman G U, Shah K, Haq F, et al., Host vector dynamics of pine wilt disease model with convex incidence rate, Chaos, Solitons \& Fractals, 2018, 113: 31-39.

[9] Zaman G, Kang Y H, and Jung I H, Optimal treatment of an SIR epidemic model with time delay, BioSystems, 2009, 98: 43-50.

[10] Khan T and Zaman G, Classification of different Hepatitis B infected individuals with saturated incidence rate, SpringerPlus, 2016, 5: 1082. 
[11] Khan A and Zaman G, Optimal control strategy of SEIR endemic model with continuous agestructure in the exposed and infectious classes, Optimal Control Applications and Methods, 2018, 39: $1716-1727$.

[12] Abdo M S, Shah K, Wahash H A, et al., On a comprehensive model of the novel coronavirus (COVID-19) under Mittag-Leffler derivative, Chaos, Solitons \& Fractals, 2020, 135: 1-14.

[13] World Health Organization, Hepatitis b fact sheet, no 204 [updated July 2015], World Health Organization, Geneva, Switzerland, 2013.

[14] Anwarud D, Li Y J, and Liu Q, Viral dynamics and control of hepatitis B virus (HBV) using an epidemic model, Alexandria Engineering Journal, 2020, 59(2): 667-679.

[15] Thornley S, Bullen C, and Roberts M, Hepatitis B in a high prevalence New Zealand population: A mathematical model applied to infection control policy, Journal of Theoretical Biology, 2008, 254: 599-603.

[16] Khan T, Zaman G, and Saleh Alshomrani A, Spreading dynamic of acute and carrier hepatitis B with nonlinear incidence, PLoS ONE, 2018, 13: e0191914.

[17] Shah K, Jarad F, and Abdeljawad T, On a nonlinear fractional order model of dengue fever disease under Caputo-Fabrizio derivative, Alexandria Engineering Journal, 2020, 59: 2305-2313.

[18] Fan M, Li M Y, and Wang K, Global stability of an SEIS epidemic model with recruitment and a varying total population size, Mathematical Biosciences, 2001, 170: 199-208.

[19] Li J and Ma Z, Qualitative analyses of SIS epidemic model with vaccination and varying total population size, Mathematical and Computer Modelling, 2002, 35: 1235-1243.

[20] Lashari A A, Hattaf K, Zaman G, et al., Backward bifurcation and optimal control of a vector borne disease, Applied Mathematics and Information Sciences, 2013, 7: 301-309.

[21] Zou L, Zhang W, and Ruan S, Modeling the transmission dynamics and control of hepatitis B virus in China, Journal of Theoretical Biology, 2010, 262: 330-338.

[22] Van den Driessche P and Watmough J, Reproduction numbers and sub-threshold endemic equilibria for compartmental models of disease transmission, Mathematical Biosciences, 2002, 180: $29-48$.

[23] Van Den Driessche P and Watmough J, Mathematical Epidemiology, Springer Verlag, New York, 2008.

[24] Koonprasert S, Moore E J, and Banyatlersthaworn S, Sensitivity and stability analysis of hepatitis $\mathrm{B}$ virus model with non-cytolytic cure process and logistic hepatocyte growth, Global Journal of Pure and Applied Mathematics, 2016, 12(3): 2297-2312.

[25] Momoh A A, Ibrahim M O, Madu B A, et al., Global equilibrium stability of hepatitis B model and vaccination impact, Research Journal of Mathematics and Statistics, 2012, 4(3): 57-62.

[26] Cheng Y, Qiuhui P, and He M, Stability analysis of hepatitis B virus model with incomplete immunization of HepB vaccine, Abstract and Applied Analysis, 2014, 1-11.

[27] Cao J, Wang Y, Alofi A, et al., Global stability of an epidemic model with carrier state in heterogeneous networks, IMA Journal of Applied Mathematics, 2015, 80(4): 1025-1048.

[28] Kamien M I and Schwartz N L, Dynamic Optimization: The Calculus of Variations and Optimal Control in Economics and Management, North Holland, Inc, 1998.

[29] Culshaw R V, Ruan S, and Spiteri R J, Optimal HIV treatment by maximising immune response, Journal of Mathematical Biology, 2004, 48: 545-562.

[30] Kirschner D, Lenhart S, and Serbin S, Optimal control of the chemotherapy of HIV, Journal of Mathematical Biology, 1997, 35: 775-792. 
[31] Suzanne L and Workman J T, Optimal Control Applied to Biological Models, Chapman and Hall/CRC, 2007.

[32] Khan T, Zaman G, and Chohan M I, The transmission dynamic and optimal control of acute and chronic hepatitis B, Journal of Biological Dynamics, 2017, 11: 172-189.

[33] Zhang J and Zhang S, Application and optimal control for an HBV model with vaccination and treatment, Discrete Dynamics in Nature and Society, 2018, Article ID 2076983, 13 pages.

[34] Kamyad A, Akbari R, Heydari A, et al., Mathematical modeling of transmission dynamics and optimal control of vaccination and treatment for hepatitis B virus, Computational and Mathematical Methods in Medicine, 2014, Article ID 475451, 15 pages.

[35] Goyal A, Murray J M, Roadmap to control HBV and HDV epidemics in China, Journal of Theoretical Biology, 2017, 423: 41-52.

[36] Pontryagin L, The Mathematical Theory of Optimal Processed, Taylor and Francis, London, UK, 1987.

[37] Nana-Kyere S, Ackora-Prah J, Okyere E, et al., Hepatitis B optimal control model with vertical transmission, Appl. Math., 2017, 7(1): 5-13.

[38] Ijalana C O and Yusuf T T, Optimal control strategy for hepatitis B virus epidemic in areas of high endemicity, International Journal of Scientific and Innovative Mathematical Research, 2017, 5(12): 28-39.

[39] Birkhoff G and Rota G, Ordinary Differential Equations, 4th Edition, John Wiley and Sons, New York, NY, USA, 1989. 